\title{
MINIMUM RANK AND ZERO FORCING NUMBER FOR BUTTERFLY NETWORKS
}

\author{
DANIELA FERRERO ${ }^{1}$, CYRIAC GRIGORIOUS ${ }^{2,3}$, THOMAS KALINOWSKI $^{2,4}$, JOE RYAN $^{2}$, AND SUDEEP STEPHEN $^{2,5}$
}

AbStract. Zero forcing is a graph propagation process introduced in quantum physics and theoretical computer science, and closely related to the minimum rank problem. The minimum rank of a graph is the smallest possible rank over all matrices described by a given network. We use this relationship to determine the minimum rank and the zero forcing number of butterfly networks, concluding they present optimal properties in regards to both problems.

\section{INTRODUCTION}

Let $G=(V, E)$ be a finite simple graph. Starting with a subset of the vertex set $V$ colored, we consider the following coloring rule: an uncolored vertex is colored if it is the only uncolored neighbor of some colored vertex. A vertex set $S \subseteq V$ is called zero-forcing if, starting with the vertices in $S$ colored and the vertices in the complement $V \backslash S$ uncolored, all the vertices can be colored by repeatedly applying the coloring rule. The minimum cardinality of a zero-forcing set for the graph $G$ is called the zero-forcing number of $G$, denoted by $Z(G)$.

Zero forcing was introduced in linear algebra to study the problem of finding the minimum rank among all symmetric matrices described by a graph [1]. Rank minimization problems consist of determining the minimum rank among all matrices whose off-diagonal zero-nonzero pattern is determined by the edges of a graph. This problem is related to the inverse eigenvalue problem [10] and with many problems in engineering involving propagation of a signal through a network [11. Minimizing the rank of a matrix is equivalent to maximizing its nullity, and the zero forcing number gives an upper bound for the maximum nullity [1]. For that reason, graphs in which the maximum nullity coincides with their zero forcing number are of special interest 1 .

Independently, the concept of zero forcing was also introduced in quantum physics, electrical engineering and theoretical computer science. In physics, zero forcing was introduced to study control of quantum systems and it is called graph infection [6, 18. The power domination problem in graph theory [13] appeared in the study of the placement of monitoring units in electrical power networks [2], and it has been proven to be equivalent to the zero forcing problem [3]. Finally, the concept of zero forcing was introduced as the fast-mixed search model for the study of fugitive search games on graphs. In fugitive search games, a group of searchers, placed on the vertices of a graph, must find a fugitive that is hiding in the vertices or edges of the graph 7 . The different games are determined by the allowed moves for the searchers and the fugitive. Depending on them, the minimum number of required searchers reveals different graph properties [4.

The fast-mixed model for graph search was introduced by Yang [19] as a combination of the fast method [9] and the mixed search method [5]. The fast-mixed number of a graph is the minimum number of searchers required to find a fugitive in the graph and it coincides with the zero forcing number.

In this paper, we prove that butterfly interconnection networks have optimal minimum rank and zero forcing properties, and as a consequence, optimal fast-search number. The interest on this particular family of graphs

\footnotetext{
${ }^{1}$ Department of Mathematics, Texas State University, San Marcos, U.S.A.

${ }^{2}$ School of Mathematical and Physical Sciences, University of Newcastle, Callaghan, Australia

${ }^{3}$ Graduate School, Kings College London, London, UK

${ }^{4}$ School of Science and Technology, University of New England, Armidale, Australia

${ }^{5}$ School of Mathematical Sciences, National Institute of Science Education and Research, Bhubaneswar, India

E-mail addresses: dferrero@txstate.edu, cyriac.grigorious@uon.edu.au, tkalinow@une.edu.au,

joe.ryan@newcastle.edu.au, sudeep.stephen@niser.ac.in.

2010 Mathematics Subject Classification. 05C96, 05C57, 94C15.

Key words and phrases. zero forcing, minimum rank of graphs, butterfly network.
} 
is that they provide an excellent model for interconnection networks where search problems are used to detect faulty nodes or false information [8, 17].

In order to make a more precise statement about the relation between the zero forcing number and the minimum rank problem, let $S_{n}(F)$ denote the set of symmetric $n \times n$ matrices over a field $F$. For a simple graph $G=(V, E)$ with vertex set $V=\{1, \ldots, n\}$, let $S(F, G)$ be the set of matrices in $S_{n}(F)$ whose non-zero off-diagonal entries correspond to edges of $G$, that is,

$$
S(F, G)=\left\{A \in S_{n}(F): i \neq j \Longrightarrow\left(i j \in E(G) \Longleftrightarrow a_{i j} \neq 0\right)\right\}
$$

The minimum $F$-rank of $G$ is defined as the minimum rank over all matrices $A$ in $S(F, G)$ :

$$
\operatorname{mr}^{F}(G)=\min \{\operatorname{rank}(A): A \in S(F, G)\} .
$$

If the index $F$ is omitted then it is understood that $F=\mathbb{R}$. The link between the zero forcing number and the minimum rank problem is established by the observation that for a zero-forcing set $S$ and a matrix $A \in S(F, G)$, the rows of $A$ that correspond to the vertices in $V \backslash S$ must be linearly independent, so $\operatorname{rank}(A) \geqslant n-|S|$, and consequently

$$
\operatorname{mr}^{F}(G) \geqslant n-Z(G) .
$$

Based on this insight, the authors of [1] determined $\operatorname{mr}(G)$ for various graph classes and established equality in (1), independent of the field $F$, in many cases. In [16, the same is proved for block-clique graphs and unit interval graphs. Recently, the zero forcing number of cartesian products of cycles was established by constructing a matrix in $S(F, G)$ with the required rank 3. The American Institute for Mathematics maintains the minimum rank graph catalog [14] in order to collect known results about the minimum rank problem for various graph classes.

The rest of the paper is structured as follows. Section 2 contains some notation and a precise statement of our main result. In Section 3, we prove an upper bound for the zero-forcing number of the butterfly network by an explicit construction of the corresponding zero forcing set $S$. By $(1)$, this implies a lower bound for the minimum rank of the butterfly network, and in Section 3 we establish that this bound is tight by showing that the rows of the adjacency matrix corresponding to the vertices in the complement of the zero-forcing set span the row space of the adjacency matrix of the butterfly network (over any field $F$ ).

\section{Notation AND MAin RESUlt}

Let $G=(V, E)$ be a finite simple graph. For a vertex $v \in V$, the open neighborhood of $v$ is the set $N(v)=$ $\{u: u v \in E(G)\}$ and the closed neighborhood of $v$ is the set $N[v]=N(v) \cup\{v\}$. We denote by $I_{n}$ the $n \times n$ identity matrix, and we use $I$ for $I_{n}$ when the order $n$ is clear from the context.

For a positive integer $r$, the butterfly network $\mathrm{BF}(r)=\left(V^{(r)}, E^{(r)}\right)$ has vertex set $V^{(r)}=V_{0}^{(r)} \cup V_{1}^{(r)} \cup \cdots \cup V_{r}^{(r)}$ and edge set $E^{(r)}=E_{1}^{(r)} \cup E_{2}^{(r)} \cup \cdots \cup E_{r}^{(r)}$, where

$$
\begin{array}{ll}
V_{i}^{(r)}=\left\{(\boldsymbol{x}, i): \boldsymbol{x} \in\{0,1\}^{r}\right\} & \text { for } i=0,1, \ldots, r, \\
E_{i}^{(r)}=\left\{\{(\boldsymbol{x}, i-1),(\boldsymbol{y}, i)\}: \boldsymbol{x} \in\{0,1\}^{r}, \boldsymbol{y} \in\left\{\boldsymbol{x}, \boldsymbol{x}+\boldsymbol{e}_{i}\right\}\right\} & \text { for } i=1,2, \ldots, r .
\end{array}
$$

Here addition is modulo 2 , and $\boldsymbol{e}_{i}$ is the binary vector of length $r$ with a one in position $i$ and zeros in all other components. For convenience, we identify the binary vector $\boldsymbol{x}=\left(x_{1}, \ldots, x_{r}\right) \in\{0,1\}^{r}$ with the number $x_{1} 2^{0}+$ $x_{2} 2^{1}+\cdots+x_{r} 2^{r-1}$. Using this identification the butterfly network $\mathrm{BF}(4)$ is shown in Figure 1 . As an example, the edges $\{(14,1),(14,2)\}$ and $\{(14,1),(12,2)\}$ in Figure 1 correspond to $\{((0,1,1,1), 1),((0,1,1,1), 2)\} \in$ $E_{2}^{(4)}$ and $\{((0,1,1,1), 1),((0,0,1,1), 2)\} \in E_{2}^{(4)}$. Note, that the order and the size of $\mathrm{BF}(r)$ are given by

$$
\left|V^{(r)}\right|=\sum_{i=0}^{r}\left|V_{i}^{(r)}\right|=(r+1) 2^{r}, \quad\left|E^{(r)}\right|=\sum_{i=1}^{r}\left|E_{i}^{(r)}\right|=r 2^{r+1} .
$$

Our main result is the following theorem.

Theorem 1. The minimum rank of the butterfly network $\mathrm{BF}(r)$ over any field $F$ equals

$$
\mathrm{mr}^{F}(\mathrm{BF}(r))=\frac{2}{9}\left[(3 r+1) 2^{r}-(-1)^{r}\right],
$$




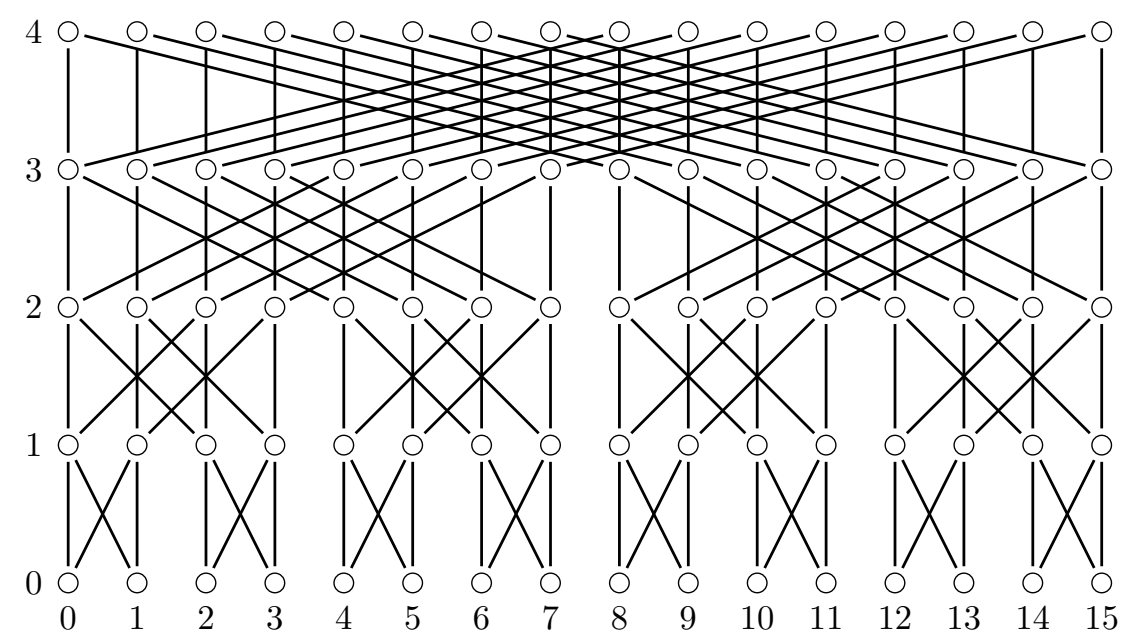

Figure 1. The butterfly network BF(4). Note the recursive structure: the bottom two rows consist of eight copies of $\mathrm{BF}(1)$, the bottom three rows of four copies of $\mathrm{BF}(2)$, and the bottom four rows of two copies of $\mathrm{BF}(3)$.

and this is equal to the rank of the adjacency matrix of $\mathrm{BF}(r)$. Furthermore, for the butterfly network we have equality in (1), i.e.,

$$
Z(\mathrm{BF}(r))=(r+1) 2^{r}-\operatorname{mr}^{F}(\mathrm{BF}(r))=\frac{1}{9}\left[(3 r+7) 2^{r}+2(-1)^{r}\right]
$$

\section{THE UPPER BOUND FOR $Z(\operatorname{BF}(r))$}

Let $\left(J_{n}\right)$ denote the Jacobsthal sequence ${ }^{1}$ which is defined by $J_{0}=0, J_{1}=1$ and $J_{n}=J_{n-1}+2 J_{n-2}$ for $n \geqslant 2$. We will need the relation

$$
J_{n+2}=2^{n}+J_{n} \quad \text { for every integer } n \geqslant 0,
$$

which can be seen as follows. We first use induction on $n$ to verify $J_{n}+J_{n+1}=2^{n}$ for all $n \geqslant 0$. The base case is $J_{0}+J_{1}=1=2^{0}$, and for $n \geqslant 1$,

$$
J_{n}+J_{n+1}=J_{n}+\left(J_{n}+2 J_{n-1}\right)=2\left(J_{n-1}+J_{n}\right)=2 \cdot 2^{n-1}=2^{n} .
$$

Then (2) follows from the recursive definition: $J_{n+2}=J_{n+1}+2 J_{n}=\left(J_{n}+J_{n+1}\right)+J_{n}=2^{n}+J_{n}$. For every $r$, we define a set $S^{(r)}=S_{0}^{(r)} \cup S_{1}^{(r)} \cup \cdots \cup S_{r}^{(r)}$ by

$$
S_{i}^{(r)}=\left\{(x, i) \in V_{i}^{(r)}: 2^{i+1} \ell \leqslant x \leqslant 2^{i+1} \ell+J_{i+1}-1 \text { for some } \ell \in\left\{0,1, \ldots,\left\lfloor\left(2^{r}-1\right) / 2^{i+1}\right\rfloor\right\}\right\}
$$

for $i=0,1, \ldots, r$. The range for $\ell$ comes from the observation that

$$
\left\lfloor\left(2^{r}-1\right) / 2^{i+1}\right\rfloor=\max \left\{\ell:\left(2^{i+1} \ell, i\right) \in V^{(r)}\right\} .
$$

In the following we will use the phrase "for some $\ell$ " as a shorthand for "for some non-negative integer $\ell$ ", and the largest relevant value of $\ell$ will always be implicit in the requirement that a certain $(x, i)$ is a vertex of $\mathrm{BF}(r)$. For $r=4$ the construction of the set $S^{(r)}$ is illustrated in Figure 2 .

Solving the recurrence relation for the numbers $J_{n}$, we find $J_{n}=\left(2^{n}-(-1)^{n}\right) / 3$, and this implies the following closed form expression for the size of the set $S^{(r)}$.

Lemma 1. We have $\left|S^{(r)}\right|=J_{r+1}+\sum_{i=1}^{r} 2^{r-i} J_{i}=\frac{1}{9}\left[(3 r+7) 2^{r}+2(-1)^{r}\right]$.

\footnotetext{
${ }^{1}$ OEIS A001045
} 


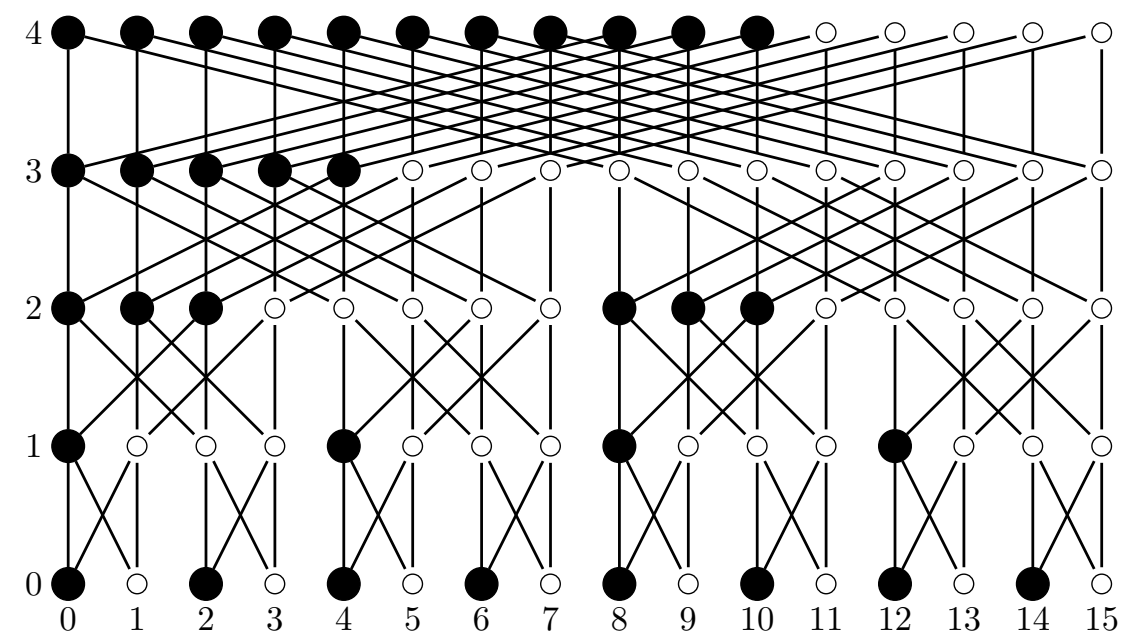

Figure 2. The set $S^{(4)}$ indicated by filled vertices.

Next we want to verify that $S^{(r)}$ is a zero forcing set for $\operatorname{BF}(r)$. For this purpose we set $X_{0}=S^{(r)}$ and define a sequence $X_{1}, X_{2}, \ldots, X_{2 r}$ of vertex sets by

$$
\begin{gathered}
X_{k}=X_{k-1} \cup\left\{(x, r-k) \in V_{r-k}^{(r)}:\{(x, r-k)\}=N(v) \backslash X_{k-1} \text { for some } v=(y, r-k+1) \in X_{k-1}\right\} \\
X_{r+k}=X_{r+k-1} \cup\left\{(x, k) \in V_{k}^{(r)}:\{(x, k)\}=N(v) \backslash X_{r+k-1} \text { for some } v=(y, k-1) \in X_{r+k-1}\right\}
\end{gathered}
$$

for $k=1, \ldots, r$. These sets can be interpreted in terms of the forcing process as follows. The sets $X_{0}$ to $X_{r}$ correspond to forcing downwards: $X_{0}$ is the set of initially colored vertices, $X_{1}$ is the set of colored vertices after the level $r$ vertices in $X_{0}$ have been used to force vertices on level $r-1, X_{2}$ is the set of colored vertices after the level $r-1$ vertices in $X_{1}$ have been used to force vertices on level $r-2$, etc., up to $X_{r}$, which is the set of colored vertices after the level 1 vertices in $X_{r-1}$ have been used to force vertices on level 0 . Then we turn around and force upwards: $X_{r+1}$ is the set of colored vertices after the level 0 vertices in $X_{r}$ have been used to force vertices on level $1, X_{r+2}$ is the set of colored vertices after the level 1 vertices in $X_{r+1}$ have been used to force vertices on level 2, etc., up to $X_{2 r}$, which is the set of colored vertices after the level $r-1$ vertices in $X_{2 r-1}$ have been used to force vertices on level $r$. As a consequence, in order to prove that $X_{0}=S^{(r)}$ is a zero forcing set, it is sufficient to prove that $X_{2 r}=V^{(r)}$. This is the purpose of the following four lemmas. Lemma 2 describes the downward forcing step from level $r-k+1$ to level $r-k$, which is then used inductively in Lemma 3 to obtain an explicit description of the sets $X_{0}$ to $X_{r}$. Similarly, Lemma 4 describes the upward forcing step from level $k-1$ to level $k$, which is then used inductively in Lemma 5 to obtain an explicit description of the sets $X_{r}$ to $X_{2 r}$.

Lemma 2. Fix $i \in\{0,1, \ldots, r-1\}$, and let $Y=Y(i) \cup Y(i+1) \cup \cdots \cup Y(r) \subseteq V^{(r)}$ be defined by

$$
\begin{array}{ll}
Y(i)=S_{i}^{(r)}=\left\{(x, i) \in V_{i}^{(r)}: 2^{i+1} \ell \leqslant x \leqslant 2^{i+1} \ell+J_{i+1}-1 \text { for some } \ell\right\} & \\
Y(j)=\left\{(x, j) \in V_{j}^{(r)}: 2^{j} \ell \leqslant x \leqslant 2^{j} \ell+J_{j+1}-1 \text { for some } \ell\right\} & \text { for } j \in\{i+1, \ldots, r\} .
\end{array}
$$

Furthermore, let $Z$ be the set of vertices on level $i$ that can be forced by vertices in $Y(i+1)$ when $Y$ is the set of colored vertices, that is, $Z=\{(x, i):\{(x, i)\}=N(v) \backslash Y$ for some $v=(y, i+1) \in Y\}$. Then

$$
Y(i) \cup Z=\left\{(x, r-k) \in V_{i}^{(r)}: 2^{i} \ell \leqslant x \leqslant 2^{i} \ell+J_{i+1}-1 \text { for some } \ell\right\} .
$$

Proof. Let $(x, i)$ be an arbitrary element of the right hand side of (5), say $2^{i} \ell \leqslant x \leqslant 2^{i} \ell+J_{i+1}-1$. We need to show that $(x, i) \in Y(i) \cup Z$. If $\ell$ is even, then $2^{i+1}(\ell / 2) \leqslant x \leqslant 2^{i+1}(\ell / 2)+J_{i+1}-1$ and $(x, i) \in S_{i}^{(r)}=Y(i)$. Otherwise, $\ell=2 \ell^{\prime}+1$ for some integer $\ell^{\prime}$ and $2^{i+1} \ell^{\prime}+2^{i} \leqslant x \leqslant 2^{i+1} \ell^{\prime}+2^{i}+J_{i+1}-1$. For $y=x-2^{i}$, the 
vertex $(y, i+1)$ is in $Y$ because

$$
2^{i+1} \ell^{\prime} \leqslant y \leqslant 2^{i+1} \ell^{\prime}+J_{i+1}-1 \leqslant 2^{i+1} \ell^{\prime}+J_{i+2}-1 .
$$

We want to show that $(y, i+1)$ forces $(x, i)$. Let $\ell^{\prime \prime}=\left\lfloor\ell^{\prime} / 2\right\rfloor$, so that $\ell^{\prime}=2 \ell^{\prime \prime}+\varepsilon$ with $\varepsilon \in\{0,1\}$. The neighborhood of $(y, i+1)$ is

$$
N((y, i+1))= \begin{cases}\{(y, i),(x, i)\} & \text { if } i=r-1 \\ \left\{(y, i),(x, i),(y, i+2),\left(y+(-1)^{\varepsilon} 2^{i+1}, i+2\right)\right\} & \text { if } i \leqslant r-2 .\end{cases}
$$

Now $(y, i) \in Y$, and for $i=r-1$ that's all we need. Using 2, we have

$$
2^{i+2} \ell^{\prime \prime}=2^{i+1}\left(\ell^{\prime}-\varepsilon\right) \leqslant 2^{i+1} \ell^{\prime} \leqslant y \leqslant 2^{i+1} \ell^{\prime}+J_{i+1}-1=2^{i+2} \ell^{\prime \prime}+2^{i+1} \varepsilon+J_{i+1}-1 \leqslant 2^{i+2} \ell^{\prime \prime}+J_{i+3}-1,
$$

and therefore $(y, i+2) \in Y$. Similarly,

$$
2^{i+2} \ell^{\prime \prime}=2^{i+1} \ell^{\prime}+(-1)^{\varepsilon} 2^{i+1} \leqslant y+(-1)^{\varepsilon} 2^{i+1} \leqslant 2^{i+2} \ell^{\prime \prime}+2^{i+1}+J_{i+1}-1 \leqslant 2^{i+2} \ell^{\prime \prime}+J_{i+3}-1,
$$

and therefore $\left(y+(-1)^{\varepsilon} 2^{i-1}, i+2\right) \in Y$. Consequently, $\{(x, i)\}=N((y, i+1)) \backslash Y$, and this implies $(x, i) \in Z$. So, we have verified

$$
Y(i) \cup Z \supseteq\left\{(x, i) \in V_{i}^{(r)}: 2^{i} \ell \leqslant x \leqslant 2^{i} \ell+J_{i+1}-1 \text { for some } \ell\right\} .
$$

To prove the other inclusion, consider any vertex $(x, i) \in V_{i}^{(r)}$ which is not contained in the right hand side of (5), that is, $2^{i} \ell+J_{i+1} \leqslant x \leqslant 2^{i}(\ell+1)-1$ for some $\ell=2 \ell^{\prime}+\varepsilon$. In particular, $(x, i) \notin Y(i)$, and it remains to be checked that $(x, i) \notin Z$, that is, $(x, i)$ cannot be forced. We have

$$
N((x, i)) \cap V_{i+1}^{(r)}=\left\{(x, i+1),\left(x+(-1)^{\varepsilon} 2^{i}, i+1\right)\right\} .
$$

If $(x, i+1) \in Y$ then

$$
\begin{aligned}
& \ell \equiv 0 \text { or } 1 \quad(\bmod 4) \Longrightarrow\left(x+2^{i+1}, i+2\right) \in N((x, i+1)) \backslash Y \text {, } \\
& \ell \equiv 2 \text { or } 3 \quad(\bmod 4) \Longrightarrow(x, i+2) \in N((x, i+1)) \backslash Y
\end{aligned}
$$

If $\left(x+(-1)^{\varepsilon} 2^{i}, i+1\right) \in Y$ then $\ell$ is odd and

$$
\begin{aligned}
& \ell \equiv 1 \quad(\bmod 4) \Longrightarrow\left(x+2^{i+1}, i+2\right) \in N((x, i+1)) \backslash Y, \\
& \ell \equiv 3 \quad(\bmod 4) \Longrightarrow(x, i+2) \in N((x, i+1)) \backslash Y \text {. }
\end{aligned}
$$

In all cases it follows that $(x, i) \notin Z$, and this concludes the proof.

Lemma 3. For $k \in\{0,1, \ldots, r\}, X_{k}=X_{k}(0) \cup X_{k}(1) \cup \cdots \cup X_{k}(r)$ with

$$
\begin{array}{ll}
X_{k}(i)=S_{i}^{(r)} & \text { for } i \in\{0,1, \ldots, r-k\} \\
X_{k}(i)=\left\{(x, i) \in V_{i}^{(r)}: 2^{i} \ell \leqslant x \leqslant 2^{i} \ell+J_{i+1}-1 \text { for some } \ell\right\} & \text { for } i \in\{r-k+1, \ldots, r\} .
\end{array}
$$

In particular, $X_{r}(0)=V_{0}^{(r)}$.

Proof. We proceed by induction on $k$. For $k=0$, there is nothing to do since $X_{0}=S^{(r)}=S_{0}^{(r)} \cup \cdots \cup S_{r}^{(r)}$. Let $k \geqslant 1$ and set $i=r-k$. By (3), the sets $X_{k}$ and $X_{k-1}$ differ only on level $i$, that is, $X_{k}(j)=X_{k-1}(j)$ for all $j \neq i$. By induction, this implies

$$
\begin{array}{ll}
X_{k}(j)=S_{j}^{(r)} & \text { for } j \in\{0,1, \ldots, i-1\} \\
X_{k}(j)=\left\{(x, j) \in V_{j}^{(r)}: 2^{j} \ell \leqslant x \leqslant 2^{j} \ell+J_{j+1}-1 \text { for some } \ell\right\} & \text { for } j \in\{i+1, \ldots, r\} .
\end{array}
$$

Also by induction,

$$
\begin{array}{ll}
X_{k-1}(j)=S_{j}^{(r)} & \text { for } j=i \\
X_{k-1}(j)=\left\{(x, j) \in V_{j}^{(r)}: 2^{j} \ell \leqslant x \leqslant 2^{j} \ell+J_{j+1}-1 \text { for some } \ell\right\} & \text { for } y \in\{i+1, \ldots, r\},
\end{array}
$$


and we can apply Lemma 2 with $Y=X_{k-1}(i) \cup X_{k-1}(i+1) \cup \cdots \cup X_{k-1}(r)$ to obtain

$$
\begin{aligned}
X_{k}(i) \stackrel{\sqrt[3]{=}}{=} X_{k-1}(i) \cup\left\{(x, i) \in V_{i}^{(r)}:\{(x, i)\}\right. & \left.=N(v) \backslash X_{k-1} \text { for some } v=(y, i+1) \in X_{k-1}\right\} \\
& =\left\{(x, i) \in V_{i}^{(r)}: 2^{i} \ell \leqslant x \leqslant 2^{i} \ell+J_{i+1}-1 \text { for some } \ell\right\} .
\end{aligned}
$$

Lemma 4. Fix $i \in\{1,2, \ldots, r\}$, and let $Y=Y(0) \cup Y(1) \cup \cdots \cup Y(i)$ be defined by

$$
\begin{array}{ll}
Y(j) & =V_{j}^{(r)} \\
Y(i) & =\left\{(x, i) \in V_{i}^{(r)}: 2^{i} \ell \leqslant x \leqslant 2^{i} \ell+J_{i+1}-1 \text { for some } \ell\right\} .
\end{array} \quad \text { for } i \in\{0,1, \ldots, i-1\},
$$

Furthermore, let $Z$ be the set of vertices on level $i$ that can be forced by vertices in $Y(i-1)=V_{i-1}^{(r)}$ when $Y$ is the set of colored vertices, that is, $Z=\{(x, i):\{(x, i)\}=N(v) \backslash Y$ for some $v=(y, i-1) \in Y\}$. Then $Y(i) \cup Z=V_{i}^{(r)}$.

Proof. Let $(x, i)$ be an arbitrary element of $V_{i}^{(r)}$. We need to show that $(x, i) \in Y(i) \cup Z$. If $2^{i} \ell \leqslant x \leqslant$ $2^{i} \ell+J_{i+1}-1$ for some $\ell$, then $(x, i) \in Y(i)$. Otherwise $2^{i} \ell+J_{i+1} \leqslant x \leqslant 2^{i}(\ell+1)-1$ for some $\ell$. Setting $y=x-2^{i-1}$, and observing that $0 \leqslant J_{i+1}-2^{i-1} \leqslant y<x \leqslant 2^{r}-1$, we obtain $(y, i-1) \in V_{i-1}^{(r)}=Y(i-1)$. The only neighbors of $(y, i-1)$ that are potentially not in $Y$ are $(x, i)$ and $(y, i)$. Using (2), we obtain

$$
2^{i} \ell \leqslant 2^{i} \ell+J_{i-1} \stackrel{2}{=} 2^{i} \ell+J_{i+1}-2^{i-1} \leqslant y \leqslant 2^{i} \ell+2^{i-1}-1 \stackrel{2 p}{=} 2^{i} \ell+J_{i+1}-J_{i-1}-1 \leqslant 2^{i} \ell+J_{i+1}-1,
$$

and therefore, $(y, i) \in Y(i)$. As a consequence $\{(x, i)\}=N((y, i-1)) \backslash Y$, which implies $(x, i) \in Z$.

Lemma 5. For $k \in\{0,1, \ldots, r\}, X_{r+k}=X_{r+k}(0) \cup X_{r+k}(1) \cup \cdots \cup X_{r+k}(r)$ with

$$
X_{r+k}(i)= \begin{cases}V_{i}^{(r)} & \text { for } i \in\{0, \ldots, k\}, \\ X_{r}(i) & \text { for } i \in\{k+1, k+2, \ldots, r\} .\end{cases}
$$

Proof. We proceed by induction on $k$. For $k=0$, there is nothing to do since $X_{r}(0)=V_{0}^{(r)}$ by Lemma 3 . For $k \geqslant 1$, the sets $X_{r+k}$ and $X_{r+k-1}$ differ only on level $k$ (see (4)), and by induction,

$$
\begin{aligned}
& X_{r+k}(i)=X_{r+k-1}(i)=V_{i}^{(r)} \\
& X_{r+k}(i)=X_{r+k-1}(i)=X_{r}(i) \\
& \text { for } i \in\{0,1, \ldots, k-1\} \\
& \text { for } i \in\{k+1, \ldots, r-1\} \text {. }
\end{aligned}
$$

We can apply Lemma 4 with $i=k$ and $Y=X_{r+k-1}(0) \cup X_{r+k-1}(1) \cup \cdots \cup X_{r+k-1}(k)$ to obtain

$$
\begin{aligned}
X_{r+k}(k) \stackrel{\text { 嵌 }}{=} X_{r+k-1}(k) \cup\left\{(x, k) \in V_{k}^{(r)}:\{(x, k)\}=N(v) \backslash X_{r+k-1} \text { for some } v=(y, k-1) \in X_{r+k-1}\right\} \\
=V_{k}^{(r)} .
\end{aligned}
$$

Combining Lemmas 3 and 5 we have proved that $S^{(r)}$ is indeed a zero forcing set for $\mathrm{BF}(r)$.

Lemma 6. For every $r \geqslant 1, S^{(r)}$ is a zero forcing set for the butterfly network $\mathrm{BF}(r)$.

From Lemmas 1 and 6 we obtain an upper bound for the zero forcing number of the butterfly network.

Proposition 1. For every $r \geqslant 1, Z(\mathrm{BF}(r)) \leqslant \frac{1}{9}\left[(3 r+7) 2^{r}+2(-1)^{r}\right]$.

\section{The LOWER BOUnd FOR $Z(\mathrm{BF}(r))$}

By (1), the corank of the adjacency matrix of a graph $G$ provides a lower bound for the zero forcing number of $G$, and consequently we can conclude the proof of Theorem 1 by establishing the following result.

Proposition 2. Let $F$ be a field, and let $A_{r}$ denote the adjacency matrix of $\mathrm{BF}(r)$ over $F$. Then

$$
\operatorname{rank}\left(A_{r}\right) \leqslant(r+1) 2^{r}-\frac{1}{9}\left[(3 r+7) 2^{r}+2(-1)^{r}\right]=\frac{2}{9}\left[(3 r+1) 2^{r}-(-1)^{r}\right] .
$$


We will prove this by verifying that the rows corresponding to vertices in $S^{(r)}$ are linear combinations of the rows corresponding to vertices in the complement of $S^{(r)}$. For this purpose, it turns out to be convenient to number the vertices recursively as indicated in Figure 3 Formally, this vertex numbering is given by a bijection $f:\{0,1,2, \ldots\}^{2} \rightarrow\{1,2,3, \ldots\}$ defined as follows. For a positive integer $x$, let $g(x)=\left\lfloor\log _{2}(x)+1\right\rfloor$, i.e., $g(x)$ is the unique integer such that $2^{g(x)-1} \leqslant x<2^{g(x)}$. In addition, let $g(0)=-1$. Then,

$$
f(x, i)= \begin{cases}i 2^{i}+x+1 & \text { if } i \geqslant g(x) \\ g(x) 2^{g(x)-1}+f\left(x-2^{g(x)-1}, i\right) & \text { if } i<g(x) .\end{cases}
$$

Note that the first argument of $f$ on the right hand side is smaller than on the left hand side, and this implies that the function $f$ is indeed well-defined by (6). For example, using $g(6)=3$ and $g(2)=2$,

$$
\begin{aligned}
& f(6,3)=3 \times 8+6+1=31, \\
& f(6,2)=3 \times 4+f(2,2)=12+2 \times 4+2+1=23, \\
& f(6,1)=3 \times 4+f(2,1)=12+2 \times 2+f(0,1)=16+1 \times 2+0+1=19 .
\end{aligned}
$$

With respect to the vertex numbering given by (6), the adjacency matrices for $\mathrm{BF}(1)$ and $\mathrm{BF}(2)$ are

$$
A_{1}=\left(\begin{array}{llll}
0 & 0 & 1 & 1 \\
0 & 0 & 1 & 1 \\
1 & 1 & 0 & 0 \\
1 & 1 & 0 & 0
\end{array}\right), \quad A_{2}=\left(\begin{array}{llll|llll|llll}
0 & 0 & 1 & 1 & 0 & 0 & 0 & 0 & 0 & 0 & 0 & 0 \\
0 & 0 & 1 & 1 & 0 & 0 & 0 & 0 & 0 & 0 & 0 & 0 \\
& 1 & 0 & 0 & 0 & 0 & 0 & 0 & 1 & 0 & 1 & 0 \\
1 & 1 & 0 & 0 & 0 & 0 & 0 & 0 & 0 & 1 & 0 & 1 \\
\hline 0 & 0 & 0 & 0 & 0 & 0 & 1 & 1 & 0 & 0 & 0 & 0 \\
0 & 0 & 0 & 0 & 0 & 0 & 1 & 1 & 0 & 0 & 0 & 0 \\
0 & 0 & 0 & 0 & 1 & 1 & 0 & 0 & 1 & 0 & 1 & 0 \\
0 & 0 & 0 & 0 & 1 & 1 & 0 & 0 & 0 & 1 & 0 & 1 \\
\hline 0 & 0 & 1 & 0 & 0 & 0 & 1 & 0 & 0 & 0 & 0 & 0 \\
0 & 0 & 0 & 1 & 0 & 0 & 0 & 1 & 0 & 0 & 0 & 0 \\
0 & 0 & 1 & 0 & 0 & 0 & 1 & 0 & 0 & 0 & 0 & 0 \\
0 & 0 & 0 & 1 & 0 & 0 & 0 & 1 & 0 & 0 & 0 & 0
\end{array}\right),
$$

and in general, $A_{r}$ has the structure illustrated in Figure 4 where $I$ is the identity matrix of size $2^{r-1} \times 2^{r-1}$.

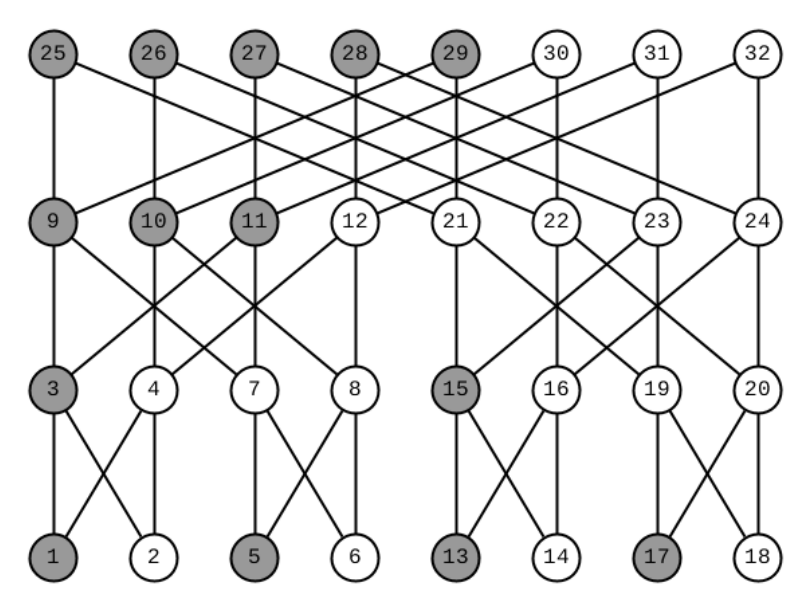

Figure 3. The butterfly network $\mathrm{BF}(3)$. The zero forcing set $S^{(3)}$ is indicated by darker vertices.

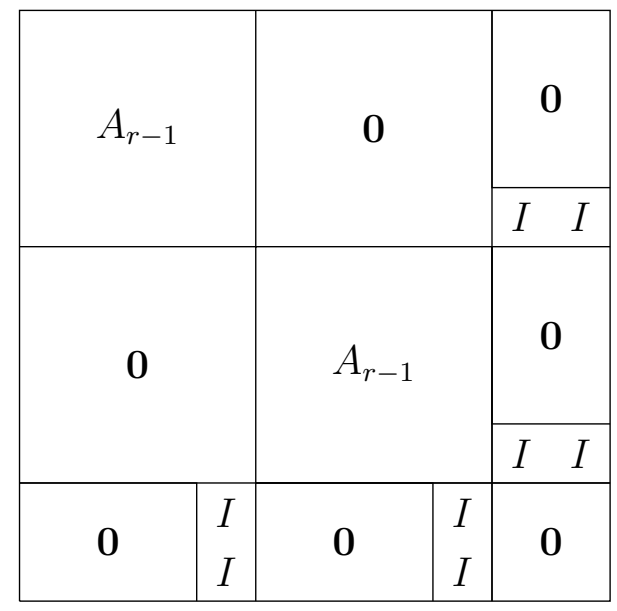

FiguRE 4. The structure of the adjacency matrix of the butterfly network $\mathrm{BF}(r)$. 
Using the vertex numbering given by $(6)$, the upper bound construction for a zero forcing set $S^{(r)} \in V(\mathrm{BF}(r))$ can be written recursively as $S^{(1)}=\{1,3\}$ and

$$
S^{(r)}=S^{(r-1)} \cup\left\{i+r 2^{r-1}: i \in S^{(r-1)}, i \leqslant(r-1) 2^{r-1}\right\} \cup\left\{r 2^{r}+1, \ldots, r 2^{r}+J_{r+1}\right\}
$$

for $r \geqslant 2$. In order to prove Proposition 2 it is sufficient to show that every row $i \in S^{(r)}$ of $A_{r}$ can be written as a linear combination of the rows in $\bar{S}^{(r)}=\left\{1, \ldots,(r+1) 2^{r}\right\} \backslash S^{(r)}$. We proceed by induction on $r$. Let $A_{r}(i)$ denote the $i$-th row of $A_{r}$. The induction base is provided by checking the cases $r=1$ and $r=2$. For $S^{(1)}=\{1,3\}$, we have

$$
\begin{aligned}
& A_{1}(1)=A_{1}(2), \\
& A_{1}(3)=A_{1}(4),
\end{aligned}
$$

and for $S^{(2)}=\{1,5,3,9,10,11\}$ (listed level by level) we have

$$
\begin{aligned}
A_{2}(1) & =A_{2}(2), \\
A_{2}(5) & =A_{2}(6), \\
A_{2}(3) & =A_{2}(4)+A_{2}(7)-A_{2}(8), \\
A_{2}(9) & =A_{2}(2)+A_{2}(6)-A_{2}(12), \\
A_{2}(10) & =A_{2}(12), \\
A_{2}(11) & =A_{2}(2)+A_{2}(6)-A_{2}(12) .
\end{aligned}
$$

The next two lemmas follow directly from the recursive structure illustrated in Figure 4.

Lemma 7. If $i \leqslant(r-1) 2^{r-1}$ and

$$
A_{r-1}(i)=\sum_{j \in K^{+}} A_{r-1}(j)-\sum_{j \in K^{-}} A_{r-1}(j) \text { for some } K^{+}, K^{-} \subseteq \bar{S}^{(r-1)},
$$

then

$$
\begin{aligned}
A_{r}(i) & =\sum_{j \in K^{+}} A_{r}(j)-\sum_{j \in K^{-}} A_{r}(j) \quad \text { and } \\
A_{r}\left(i+r 2^{r-1}\right) & =\sum_{j \in K^{\prime+}} A_{r}(j)-\sum_{j \in K^{\prime-}} A_{r}(j)
\end{aligned}
$$

where $K^{+}, K^{-} \subseteq \bar{S}^{(r)}$ and $K^{\prime \varepsilon}=\left\{j+r 2^{r-1}: j \in K^{\varepsilon}\right\} \subseteq \bar{S}^{(r)}$ for $\varepsilon \in\{+,-\}$.

Lemma 8. If $(r-1) 2^{r-1}+1 \leqslant i \leqslant(r-1) 2^{r-1}+J_{r}$ and

$$
A_{r-1}(i)=\sum_{j \in K^{+}} A_{r-1}(j)-\sum_{j \in K^{-}} A_{r-1}(j) \text { for some } K^{+}, K^{-} \subseteq \bar{S}^{(r-1)},
$$

then

$$
A_{r}(i)=\sum_{j \in K^{\prime+}} A_{r}(j)-\sum_{j \in K^{\prime-}} A_{r}(j)
$$

where

$$
\begin{aligned}
& K^{\prime+}=K^{+} \cup\left\{j+r 2^{r-1} \quad: j \in K^{-}\right\} \cup\left\{i+r 2^{r-1}\right\} \subseteq \bar{S}^{(r)} \\
& K^{\prime-}=K^{-} \cup\left\{j+r 2^{r-1}: j \in K^{+}\right\} \subseteq \bar{S}^{(r)}
\end{aligned}
$$

To illustrate the step from $A_{r-1}(i)$ to $A_{r}(i)$ in Lemma 8, consider $r=3$ and $i=10$. From (14), we have $K^{+}=\{12\}$ and $K^{-}=\emptyset$, and by Lemma 8 (note that $r 2^{r-1}=12$ ), $K^{\prime+}=\{12,22\}$ and $K^{\prime-}=\{24\}$, hence 
$A_{3}(10)=A_{3}(12)+A_{3}(22)-A_{3}(24)$. This can be seen in terms of the recursive structure indicated in Figure 4 as follows. We have $A_{2}(10)=A_{2}(12)=\boldsymbol{e}_{4}+\boldsymbol{e}_{8}$, and then,

$$
\begin{array}{ll}
A_{3}(10)=\boldsymbol{e}_{4}+\boldsymbol{e}_{8}+\boldsymbol{e}_{26}+\boldsymbol{e}_{30}, & A_{3}(12)=\boldsymbol{e}_{4}+\boldsymbol{e}_{8}+\boldsymbol{e}_{28}+\boldsymbol{e}_{32}, \\
A_{3}(22)=\boldsymbol{e}_{16}+\boldsymbol{e}_{20}+\boldsymbol{e}_{26}+\boldsymbol{e}_{30}, & A_{3}(24)=\boldsymbol{e}_{16}+\boldsymbol{e}_{20}+\boldsymbol{e}_{28}+\boldsymbol{e}_{32} .
\end{array}
$$

Lemmas 7 and 8 take care of the first two components in the recursion for $S^{(r)}$ in (7). It remains to check the rows $r 2^{r}+i$ for $i \in\left\{1, \ldots, J_{r+1}\right\}$. For $J_{r-1}+1 \leqslant i \leqslant 2^{r-1}$ the required linear dependence is $A_{r}\left(r 2^{r}+i\right)=$ $A_{r}\left(r 2^{r}+i+2^{r-1}\right)$, because $i+2^{r-1}>J_{r+1}$ and therefore $r 2^{r}+i+2^{r-1} \in \bar{S}^{(r)}$. For $i>2^{r-1}$ we have $i \leqslant 2^{r-1}+J_{r-1}$ and $A_{r}\left(r 2^{r}+i\right)=A_{r}\left(r 2^{r}+i-2^{r-1}\right)$, and consequently it is sufficient to consider $i \in$ $\left\{1, \ldots, J_{r-1}\right\}$. The induction step for these cases will be from $\operatorname{BF}(r-2)$ to $\operatorname{BF}(r)$, so we have to take the recursion for the adjacency matrix one step further which is illustrated in Figure 5 . The basic idea is as follows.

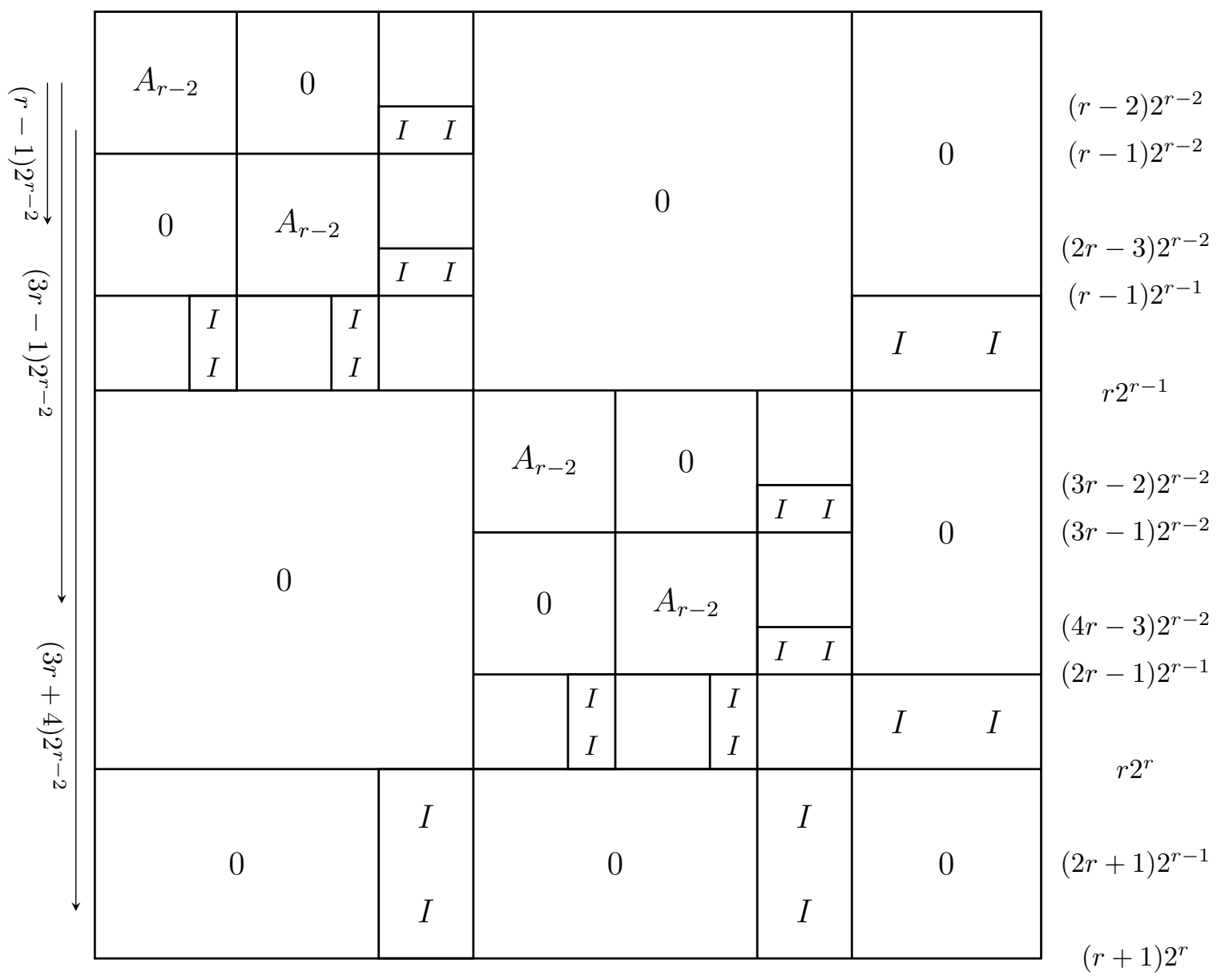

FiguRE 5. The second level of the recursion for $A_{r}$.

Let $i \in\left\{1, \ldots, J_{r-1}\right\}$. Then $(r-2) 2^{r-2}+i \in S^{(r-2)}$, and by induction there are sets $K^{+}, K^{-} \subseteq \bar{S}^{(r-2)}$ such that

or equivalently

$$
A_{r-2}\left((r-2) 2^{r-2}+i\right)=\sum_{j \in K^{+}} A_{r-2}(j)-\sum_{j \in K^{-}} A_{r-2}(j)
$$

$$
\sum_{j \in K^{+}} A_{r-2}(j)-\sum_{j \in K^{\prime-}} A_{r-2}(j)=\mathbf{0}
$$


where $K^{\prime-}=K^{-} \cup\left\{(r-2) 2^{r-2}+i\right\}$. This is a linear dependence of the rows of $A_{r-2}$ with coefficients in $\{1,-1\}$ and involving exactly one of the rows $(r-2) 2^{r-2}+1, \ldots,(r-2) 2^{r-2}+J_{r-1}$, namely $(r-2) 2^{r-2}+i$. Putting $K=K^{+} \cup K^{\prime-}$ we have

$$
K \cap\left\{(r-2) 2^{r-2}+1, \ldots,(r-2) 2^{r-2}+J_{r-1}\right\}=\left\{(r-2) 2^{r-2}+i\right\} .
$$

We now translate the $|K|$ rows in this linear dependence by $(r-1) 2^{r-2}$ and $(3 r-1) 2^{r-2}$ as indicated in Figure 5 The combination of the $2|K|$ translated rows is a $\{0,1,-1\}$-vector $\boldsymbol{x}$ which has all its nonzero entries in columns with indices in $\left\{(r-1) 2^{r-1}+1, \ldots, r 2^{r-1}\right\} \cup\left\{(2 r-1) 2^{r-1}+1, \ldots, r 2^{r}\right\}$, and has $x_{k}=1$ for $k \in\left\{(r-1) 2^{r-1}+i,(2 r-1) 2^{r-1}+i\right\}$ which are the one-entries of the row $A_{r}\left(r 2^{r}+i\right)$. Finally, we use some of the rows $r 2^{r}+J_{r+1}+1, \ldots,(r+1) 2^{r}$ with the appropriate sign to eliminate the other nonzero entries of $\boldsymbol{x}$.

More precisely, we define $\tilde{K}=\tilde{K}^{+} \cup \tilde{K}^{-} \subseteq\left\{1, \ldots,(r+1) 2^{r}\right\}$ with $\tilde{K}^{+}=\tilde{K}_{1}^{+} \cup \tilde{K}_{2}^{+}$and $\tilde{K}^{-}=\tilde{K}_{1}^{-} \cup \tilde{K}_{2}^{-}$ where

$$
\begin{aligned}
& \tilde{K}_{1}^{+}=\left\{j+(r-1) 2^{r-2}: j \in K^{\prime-}\right\} \cup\left\{j+(3 r-1) 2^{r-2}: j \in K^{\prime-}\right\} \\
& \tilde{K}_{1}^{-}=\left\{j+(r-1) 2^{r-2}: j \in K^{+}\right\} \cup\left\{j+(3 r-1) 2^{r-2}: j \in K^{+}\right\} \\
& \tilde{K}_{2}^{+}=\left\{j+(3 r+4) 2^{r-2}: j \in K^{+} \text {with } j>(r-2) 2^{r-2}\right\} \\
& \cup\left\{j+(3 r+5) 2^{r-2}: j \in K^{+} \text {with } j>(r-2) 2^{r-2}\right\} . \\
& \tilde{K}_{2}^{-}=\left\{j+(3 r+4) 2^{r-2}: j \in K^{-} \text {with } j>(r-2) 2^{r-2}\right\} \\
& \cup\left\{j+(3 r+5) 2^{r-2}: j \in K^{-} \text {with } j>(r-2) 2^{r-2}\right\} .
\end{aligned}
$$

The construction of $\tilde{K}$ is illustrated for $r=4$ and $i=1$ in Figure 6. Here, we want to construct a linear

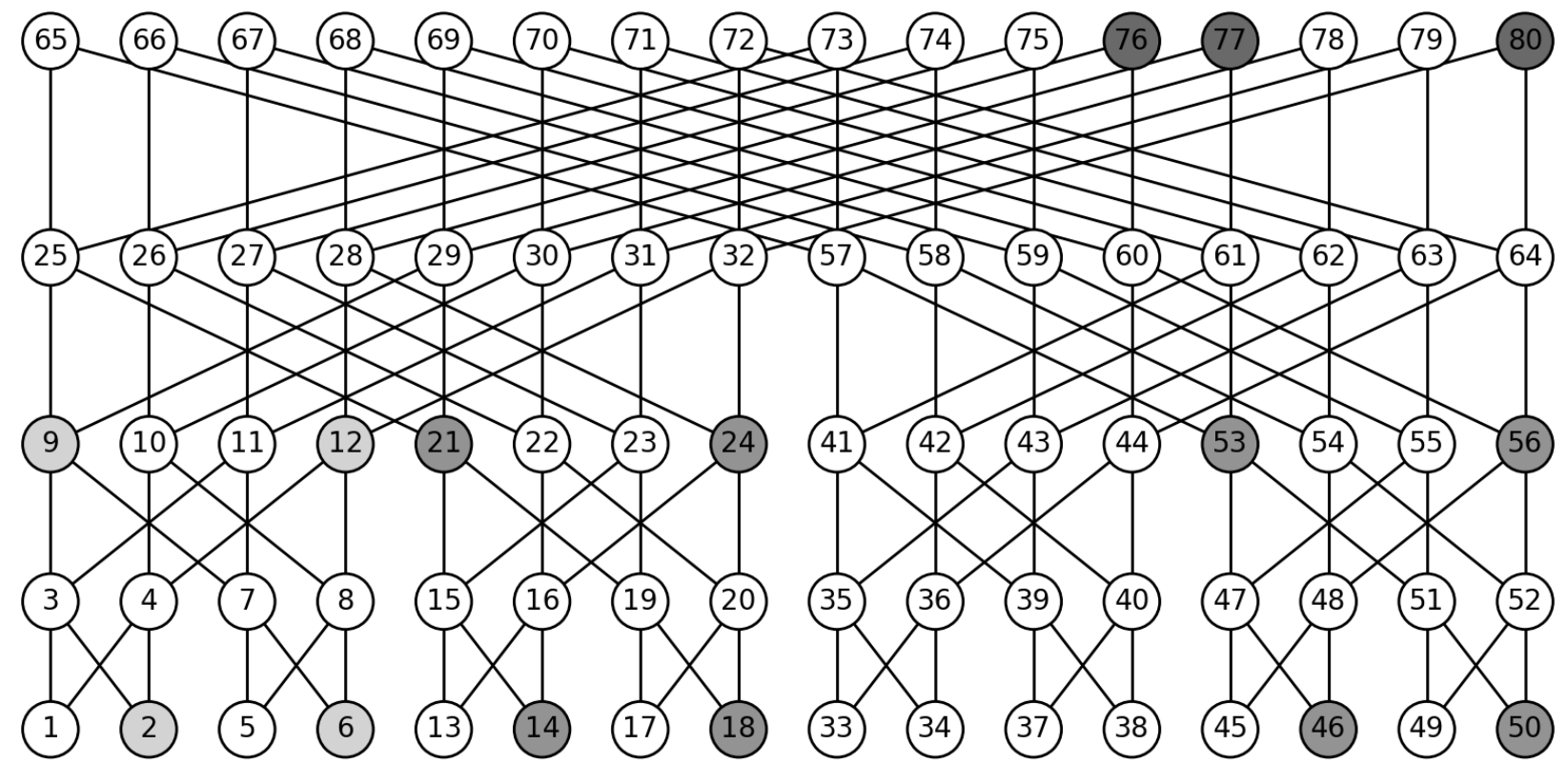

Figure 6. The construction of $\tilde{K}$ for $r=4$ and $i=1$. Here $K^{+}=\{2,6\}, K^{--}=\{9,12\}$, $\tilde{K}_{1}^{-}=\{14,18,46,50\}, \tilde{K}_{1}^{+}=\{21,24,53,56\}, \tilde{K}_{2}^{-}=\{76,77,80\}, \tilde{K}_{2}^{+}=\emptyset$.

combination representing $A_{4}(65)$, and we start with $(13)$ in the form $A_{2}(2)+A_{2}(6)-A_{2}(9)-A_{2}(12)=\mathbf{0}$ with $K^{+}=\{2,6\}$ and $K^{\prime-}=\{9,12\}$. Shifting by 12 and 44 , we obtain $\tilde{K}^{-}=\{14,18,46,50\}$ and $\tilde{K}^{+}=$ 
$\{21,24,53,56\}$, and looking at Figure 5, we can use $A_{2}(2)+A_{2}(6)-A_{2}(9)-A_{2}(12)=\mathbf{0}$ to verify that

$$
\begin{aligned}
A_{4}(21)+A_{4}(24)+A_{4}(53)+A_{4}(56)-A_{4}(14)-A_{4}(18) & -A_{4}(46)-A_{4}(50) \\
& =e_{25}+e_{28}+e_{29}+e_{32}+e_{57}+e_{60}+e_{61}+e_{64}
\end{aligned}
$$

where the right hand side corresponds to the (level 3)-neighborhood of $\{21,24,53,56\}$ which is the set of indices coming from shifting 9 and 12. The contributions of rows from shifting 2 and 6 cancel, because these rows do not see the identity matrices in columns 25 to 32 (see Figure 4), which reflects the fact that vertices 2 and 6 don't have neighbors on level 3. Now we note that the neighborhood of $\{76,77,80\}$ is $\{28,29,32,60,61,64\}$, and conclude

$$
\begin{array}{r}
A_{4}(21)+A_{4}(24)+A_{4}(53)+A_{4}(56)-A_{4}(14)-A_{4}(18)-A_{4}(46)-A_{4}(50)-A_{4}(76)-A_{4}(77)-A_{4}(80) \\
=e_{25}+e_{57}=A_{4}(65)
\end{array}
$$

as required.

Lemmas 9 and 10 state that $\tilde{K}$ has the required properties.

Lemma 9. Let $r \geqslant 3, i \in\left\{1, \ldots, J_{r-1}\right\}$, suppose $K \subseteq \bar{S}^{(r-2)}$ satisfies [16), and define $\tilde{K}$ by (19) to 22). Then $\tilde{K} \subseteq \bar{S}^{(r)}$.

Proof. Note that by construction

$$
\tilde{K}_{1}=\tilde{K}_{1}^{+} \cup \tilde{K}_{1}^{-} \subseteq\left\{(r-1) 2^{r-2}+1, \ldots,(r-1) 2^{r-1}\right\} \cup\left\{(3 r-1) 2^{r-2}+1, \ldots,(2 r-1) 2^{r-1}\right\} .
$$

Suppose there is an element $j \in K$ such that $k=j+(r-1) 2^{r-2} \in \tilde{K}_{1} \cap S^{(r)}$. From $j \in K \subseteq V^{(r-2)}$ it follows that $k \leqslant(r-1) 2^{r-2}+(r-1) 2^{r-2}=(r-1) 2^{r-1}<r 2^{r-1}$. Using (7), we obtain

$$
\begin{aligned}
k \in S^{(r)}=S^{(r-1)} \cup\left\{p+r 2^{r-1}: p \in S^{(r-1)}, p \leqslant(r-1) 2^{r-1}\right\} \cup\left\{r 2^{r}+1, \ldots, r 2^{r}+J_{r+1}\right\} \\
\stackrel{k r 2^{r-1}}{\Longrightarrow} k \in S^{(r-1)}=S^{(r-2)} \cup\left\{p+(r-1) 2^{r-2}: p \in S^{(r-2)}, p \leqslant(r-2) 2^{r-2}\right\} \\
\cup\left\{(r-1) 2^{r-2}+1, \ldots,(r-1) 2^{r-1}+J_{r}\right\} \\
\Longrightarrow k=p+(r-1) 2^{r-2} \text { for some } p \in S^{(r-2)} \text { with } p \leqslant(r-2) 2^{r-2},
\end{aligned}
$$

which contradicts the assumption that $j \in K \subseteq \bar{S}^{(r-2)} \cup\left\{(r-2) 2^{r-2}+i\right\}$. Similarly, for $k=j+(3 r-1) 2^{r-1} \in$ $\tilde{K}_{1} \cap S^{(r)}$ we obtain

$$
\begin{aligned}
& k \in S^{(r)}=S^{(r-1)} \cup\left\{p+r 2^{r-1}: p \in S^{(r-1)}, p \leqslant(r-1) 2^{r-1}\right\} \cup\left\{r 2^{r}+1, \ldots, r 2^{r}+J_{r+1}\right\} \\
\Longrightarrow & k=p+r 2^{r-1} \text { for some } p \in S^{(r-1)} \text { with } p \leqslant(r-1) 2^{r-1} \\
\Longrightarrow & k=q+(r-1) 2^{r-2} \text { for some } q \in S^{(r-2)} \text { with } q \leqslant(r-2) 2^{r-2},
\end{aligned}
$$

where we use $k>(3 r-1) 2^{r-2}$ for the last implication. Again we obtain a contradiction to the assumption that $j \in K \subseteq \bar{S}^{(r-2)} \cup\left\{(r-2) 2^{r-2}+i\right\}$. Finally, the elements of $\tilde{K}_{2}=\tilde{K}_{2}^{+} \cup \tilde{K}_{2}^{-}$are in $\bar{S}^{(r)}$ since for $j \in K^{+} \cup K^{-}$ we have

$$
j>(r-2) 2^{r-2} \Longrightarrow j>(r-2) 2^{r-2}+J_{r-1} \Longrightarrow j+(3 r+4) 2^{r-2}>r 2^{r}+2^{r-1}+J_{r-1}=2^{r}+J_{r+1},
$$

and for $j \in K^{\prime}$,

$$
j>(r-2) 2^{r-2} \Longrightarrow j+(3 r+5) 2^{r-2}>r 2^{r}+2^{r-1}+2^{r-2}>r 2^{r}+J_{r+1},
$$

and this concludes the proof of the lemma.

Lemma 10. Let $r \geqslant 3, i \in\left\{1, \ldots, J_{r-1}\right\}$, suppose $K^{+}, K^{-} \subseteq \bar{S}^{(r-2)}$ satisfy $\left[16\right.$, and define $\tilde{K}^{+}$and $\tilde{K}^{-}$ by 19 to 22 . Then

$$
A_{r}\left(r 2^{r}+i\right)=\sum_{j \in \tilde{K}^{+}} A_{r}(j)-\sum_{j \in \tilde{K}^{-}} A_{r}(j) .
$$


Proof. Setting

$$
\boldsymbol{x}=\sum_{j \in \tilde{K}_{1}^{+}} A_{r}(j)-\sum_{j \in \tilde{K}_{1}^{-}} A_{r}(j), \quad \quad \boldsymbol{y}=A_{r}\left(r 2^{r}+i\right)-\sum_{j \in \tilde{K}_{2}^{+}} A_{r}(j)+\sum_{j \in \tilde{K}_{2}^{-}} A_{r}(j)
$$

equation $(24)$ is equivalent to $\boldsymbol{x}=\boldsymbol{y}$. From 23 and 16 it follows that

$$
\operatorname{supp}(\boldsymbol{x}) \subseteq\left\{(r-1) 2^{r-1}+1, \ldots, r 2^{r-1}\right\} \cup\left\{(2 r-1) 2^{r-1}+1, \ldots, r 2^{r}\right\},
$$

and by construction, for every $j \in\left\{1, \ldots, 2^{r-2}\right\}$,

$$
\boldsymbol{x}\left((r-1) 2^{r-1}+j\right)=\boldsymbol{x}\left((r-1) 2^{r-1}+2^{r-2}+j\right)=\boldsymbol{x}\left((2 r-1) 2^{r-1}+j\right)=x\left((2 r-1) 2^{r-1}+2^{r-2}+j\right) .
$$

Denoting this value by $\tilde{x}(j)$, we have

$$
\tilde{\boldsymbol{x}}(j)= \begin{cases}1 & \text { if }(r-2) 2^{r-2}+j \in K^{\prime-}, \\ -1 & \text { if }(r-2) 2^{r-2}+j \in K^{+}, \\ 0 & \text { otherwise. }\end{cases}
$$

From 21 and 22 it follows that

$$
\tilde{K}_{2}^{+} \cup \tilde{K}_{2}^{-} \cup\left\{(2 r+1) 2^{r-1}+i\right\} \subseteq\left\{(2 r+1) 2^{r+1}+1, \ldots,(r+1) 2^{r}\right\},
$$

and therefore

$$
\operatorname{supp}(\boldsymbol{y}) \subseteq\left\{(r-1) 2^{r-1}+1, \ldots, r 2^{r-1}\right\} \cup\left\{(2 r-1) 2^{r-1}+1, \ldots, r 2^{r}\right\},
$$

After replacing $A_{r}\left(r 2^{r}+i\right)$ by $A_{r}\left(r 2^{r}+2^{r-1}+i\right)$ (which we can do since the two rows are equal), the rows contributing to $\boldsymbol{y}$ come in pairs $\left(j, j+2^{r-2}\right)$ where both rows in each pair have the same sign in $\boldsymbol{y}$. Therefore,

$$
\boldsymbol{y}\left((r-1) 2^{r-1}+j\right)=y\left((r-1) 2^{r-1}+2^{r-2}+j\right)=\boldsymbol{y}\left((2 r-1) 2^{r-1}+j\right)=\boldsymbol{y}\left((2 r-1) 2^{r-1}+2^{r-2}+j\right) .
$$

For $j \in\left\{1, \ldots, 2^{r-2}\right\}$ we have $\boldsymbol{y}\left((r-1) 2^{r-1}+j\right)=1$ if and only if $(2 r+1) 2^{r-1}+j=j^{\prime}+(3 r+4) 2^{r-1}$ for some $j^{\prime} \in K^{\prime-}$, or equivalently $j^{\prime}=(r-2) 2^{r-2}+j \in K^{\prime-}$. Similarly, we have $\boldsymbol{y}\left((r-1) 2^{r-1}+j\right)=-1$ if and only if $j^{\prime}=(r-2) 2^{r-2}+j \in K^{+}$, and comparing this with 25) we conclude $\boldsymbol{x}=\boldsymbol{y}$, as required.

Proof of Proposition 1. The statement follows by induction with base (8)-(15), using Lemmas 7, 8,9 and 10 for the induction step.

Finally, Theorem 1 is a consequence of Propositions 1 and 2.

\section{Additional COMments}

For a graph $G=(V, E)$ and a zero-forcing set $S \subseteq V$, the propagation time pt $(S)$ has been defined in [15] as the length $m$ of the increasing sequence $S=S_{0} \subsetneq S_{1} \subseteq \cdots \subsetneq S_{m}=V$, where

$$
S_{i}=S_{i-1} \cup\left\{w:\{w\}=N(v) \cap S_{i-1} \text { for some } v \in S_{i-1}\right\} \quad \text { for } i=1,2 \ldots
$$

The propagation time of $\operatorname{pt}(G)$ of the graph $G$ is the minimum of the propagation times $\operatorname{pt}(S)$ over all minimum zero-forcing sets $S$. The construction in Section 3 gives the upper bound $\operatorname{pt}(\mathrm{BF}(r)) \leqslant 2 r$, and we leave it as an open problem to determine the propagation time of $\mathrm{BF}(r)$. As mentioned in the introduction, a concept closely related to zero-forcing, called power domination, was introduced in [13. A vertex set $S \subseteq V$ is called power dominating if the closed neighborhood $N[S]=S \cup\{w: v w \in E$ for some $v \in S\}$ is a zero forcing set. It was shown in [3] that $Z(G) / \Delta$ provides a lower bound for the size of a power dominating set in $G$ where $\Delta$ is the maximum degree of $G$. This implies that the power domination number of the butterfly network $\mathrm{BF}(r)$, that is, the minimum size of a power dominating set, is at least

$$
\left\lceil\frac{1}{36}\left[(3 r+7) 2^{r}+2(-1)^{r}\right]\right\rceil .
$$

This bound does not appear to be tight and we leave for future work the problems of finding the power domination number of the butterfly network as well as its power propagation time which is defined in [12] as

$$
\operatorname{ppt}(G)=1+\min \{\operatorname{pt}(N[S]): S \text { is a minimum power dominating set in } G\} \text {. }
$$


Acknowledgement. We would like to thank an anonymous reviewer for carefully reading a previous version of the paper and providing a large number of insightful comments which were incredibly helpful in clarifying the presentation of our arguments.

\section{References}

[1] AIM Minimum Rank - Special Graphs Work Group (F. Barioli, W. Barrett, S. Butle, S. M. Cioabă, D. Cvetković, S. M. Fallat, C. Godsil, W. Haemers, L. Hogben, R. Mikkelson, S. Narayan, O. Pryporova, I. Sciriha, W. So, D. Stevanović, H. van der Holst, K. Van der Meulen, A. Wangsness), Zero forcing sets and the minimum rank of graphs, Linear Algebra and its Applications 428 (2008), no. 7, 1628-1648.

[2] Thomas L. Baldwin, Lamine Mili, Monte B. Boisen, and Ram Adapa, Power system observability with minimal phasor measurement placement, IEEE Transactions on Power Systems 8 (1993), no. 2, 707-715.

[3] Katherine F. Benson, Daniela Ferrero, Mary Flagg, Veronica Furst, Leslie Hogben, Violeta Vasilevska, and Brian Wissman, Zero forcing and power domination for graph products, Australasian Journal of Combinatorics 70 (2017), no. 2, $221-235$.

[4] Daniel Bienstock, Graph searching, path-width, tree-width and related problems (a survey), Reliability of computer and communication networks (Fred Stephen Roberts, Frank K. Hwang, and Clyde L. Monma, eds.), DIMACS Series in Discrete Mathematics and Theoretical Computer Science, vol. 5, 1991, pp. 33-49.

[5] Daniel Bienstock and Paul Seymour, Monotonicity in graph searching, Journal of Algorithms 12 (1991), no. 2, $239-245$.

[6] Daniel Burgarth and Vittorio Giovannetti, Full control by locally induced relaxation, Physical Review Letters 99 (2007), no. 10, 100501.

[7] Nick D. Dendris, Lefteris M. Kirousis, and Dimitrios M. Thilikos, Fugitive-search games on graphs and related parameters, Theoretical Computer Science 172 (1997), no. 1-2, 233-254.

[8] Stefan Dobrev, Paola Flocchini, Rastislav Královič, Peter Ružička, Giuseppe Prencipe, and Nicola Santoro, Black hole search in common interconnection networks, Networks 47 (2006), no. 2, 61-71.

[9] Danny Dyer, Boting Yang, and Öznur Yaşar, On the fast searching problem, Algorithmic Aspects in Information and Management, Lecture Notes in Computer Science, Springer Berlin Heidelberg, 2008, pp. 143-154.

[10] Shaun M. Fallat and Leslie Hogben, The minimum rank of symmetric matrices described by a graph: A survey, Linear Algebra and its Applications 426 (2007), no. 2-3, 558-582.

[11] Maryam Fazel, Haitham Hindi, and Stephen Boyd, Rank minimization and applications in system theory, Proceedings of the 2004 American Control Conference, vol. 4, IEEE, 2004, pp. 3273-3278.

[12] Daniela Ferrero, Leslie Hogben, Franklin H. J. Kenter, and Michael Young, Note on power propagation time and lower bounds for the power domination number, Journal of Combinatorial Optimization 34 (2016), no. 3, $736-741$.

[13] Teresa W. Haynes, Sandra M. Hedetniemi, Stephen T. Hedetniemi, and Michael A. Henning, Domination in graphs applied to electric power networks, SIAM J. Discrete Math. 15 (2002), no. 4, 519-529.

[14] Leslie Hogben, Wayne Barrett, Jason Grout, Hein van der Holst, Kaela Rasmussen, and Andrew Smith., AIM minimum rank graph catalog, 2016, http://admin.aimath.org/resources/graph-invariants/minimumrankoffamilies/\#/cuig

[15] Leslie Hogben, My Huynh, Nicole Kingsley, Sarah Meyer, Shanise Walker, and Michael Young, Propagation time for zero forcing on a graph, Discrete Applied Mathematics 160 (2012), no. 13-14, 1994-2005.

[16] Liang-Hao Huang, Gerard J. Chang, and Hong-Gwa Yeh, On minimum rank and zero forcing sets of a graph, Linear Algebra and its Applications 432 (2010), no. 11, 2961-2973.

[17] Lefteris M. Kirousis, Evangelos Kranakis, Danny Krizanc, and Yannis C. Stamatiou, Locating information with uncertainty in fully interconnected networks, International Symposium on Distributed Computing, Lecture Notes in Computer Science, vol. 1914, Springer Berlin Heidelberg, 2000, pp. 283-296.

[18] Simone Severini, Nondiscriminatory propagation on trees, Journal of Physics A: Mathematical and Theoretical 41 (2008), no. 48, 482002 .

[19] Boting Yang, Fast-mixed searching and related problems on graphs, Theoretical Computer Science 507 (2013), $100-113$. 